\title{
REVIEW
}

\section{SARS-CoV-2 and COVID-19: How much do we know?}

\author{
M. WANG ${ }^{1,2,3}$, Z. LIAO ${ }^{1,4^{*}}$
}

\begin{abstract}
${ }^{1}$ School of Biological Sciences, Nanyang Technological University, 60 Nanyang Drive, Singapore 637551, Singapore; ${ }^{2}$ Department of Acupuncture and Moxibustion, Beijing Hospital of Traditional Chinese Medicine, Capital Medical University, Beijing Key Laboratory of Acupuncture Neuromodulation, Beijing 100010, P. R. China; ${ }^{3}$ Graduate School, Beijing University of Chinese Medicine, Beijing 100029, P. R. China; ${ }^{4}$ Department of Microbiology, Tumor and Cell Biology (MTC), Karolinska Institutet, Biomedicum, Solnavägen 9, Stockholm 17177, Sweden
\end{abstract}

Received May 27, 2020; accepted June 4, 2020

Summary.-The recent outbreak of coronavirus disease (COVID-19) globally threatens the public health. COVID-19 is a pneumonia caused by severe acute respiratory syndrome coronavirus 2 (SARS-CoV-2), previously known as the 2019 novel coronavirus (2019-nCoV). Typical symptoms of COVID-19 include fever, cough and fatigue. As a novel disease, there are still many unsolved questions regarding COVID-19. Nevertheless, genetic analysis has demonstrated that the virus is strongly associated with certain SARSlike coronavirus originated from bats. The COVID-19 outbreak started in a seafood wholesale market in Wuhan, China, but the exact origin of the virus is still highly debatable. Since there is currently no registered antiviral drug for the disease, symptomatic treatments have been applied routinely to manage COVID-19 cases. However, various drugs and vaccines have been currently under research. This review aims to consolidate and discuss the likely origins and genetic features of SARS-CoV-2 as well as the recent clinical findings and potential effective treatments of COVID-19.

Keywords: COVID-19; SARS-CoV-2; 2019-nCoV; SARS; coronavirus

\section{Introduction}

In December 2019, several local health facilities in Wuhan, Hubei, China, reported a series of unexplained cases of pneumonia, of which the clinical presentations were extremely similar to viral pneumonia (Cohen and Nor-

"Corresponding author. E-mail: liao0058@e.ntu.edu.sg; phone: +65-97622986.

Abbreviations: ARDS = acute respiratory distress syndrome; COVID-19 = coronavirus disease 2019; ICU = intensive care unit; MERS = Middle East respiratory syndrome coronavirus; $\mathrm{RBD}=$ receptor-binding domain; SARS = severe acute respiratory syndrome; SARS-CoV-2 = SARS coronavirus 2; $\mathrm{WHO}=$ World Health Organization; 2019-nCoV = 2019 novel coronavirus mile, 2020). Later, at the end of December 2019, the Health Commission of Hubei province, China, first proclaimed 27 patients with unknown cause of pneumonia. On the same day, the Chinese Centre for Disease Control and Prevention (China CDC) dispatched a cluster of experts to Hubei province to help local health authorities to investigate the prevalence and cause of the unknown pneumonia (Centre for Health Protection of the Hong Kong, 2020). Then, on $7^{\text {th }}$ January 2020, a novel coronavirus was first detected by China CDC from the throat swab specimen of a patient, and World Health Organization (WHO) named it 2019 novel coronavirus (2019-nCoV) five days later. On $11^{\text {th }}$ February 2020, according to the agreed guidelines of WHO about assignment of disease names which are not supposed to contain a geographical location, an animal, an individual or group of people, but should represent the 
disease, the novel coronavirus pneumonia was officially renamed COVID-19 meaning coronavirus disease 2019 (WHO, 2020a).

The current outbreak of COVID-19 reminds us of two other similar coronaviruses which emerged as major global health threats as well. Firstly, it was the severe acute respiratory syndrome coronavirus (SARS-CoV), which first appeared in southern China, and lasted approximately 8 months during years 2002 to 2003 . The outbreak of SARS-CoV spread to 37 countries and eventually led to more than 8000 infections and 800 deaths (Zhong et al., 2003; Ksiazek, 2003). Secondly, the Middle East respiratory syndrome coronavirus (MERS-CoV), which was first identified in Saudi Arabia in 2012. Twenty-seven countries have reported cases of MERS-CoV since September 2012. To date, MERS-CoV has infected 2494 individuals and caused 858 deaths worldwide according to the data from WHO (WHO, 2020a; Zaki et al., 2012; Wu et al., 2020). It is worth noting that both SARS-CoV and MERS-CoV were found to have super spreaders, by which a single individual case can infect more than 100 individuals. So far, there is no super spreader of COVID-19 found in China. However, it has already contributed to hundreds of thousands of infections.

The basic reproductive number $\left(R_{0}\right)$ is generally applied to evaluate the transmissibility of a virus. Previous studies have already revealed that the estimate of $R_{0}$ for SARS-CoV and MERS-CoV is about 3 and 1, respectively (Bauch et al., 2005; Romulus et al., 2013). However, there is no consensus of $R_{0}$ for COVID-19, although several estimates have been done. Studies using stochastic methods indicate that $\mathrm{R}_{0}$ are 2.2 and 2.68 (Li et al., 2020; Wu et al., 2020), which are lower than SARS-CoV. However, a study using both stochastic and statistical methods, estimate $\mathrm{R}_{0}$ as 3.28, which is higher than SARS-CoV (Liu et al., 2020). Although the results are not the same, COVID-19 causes more infections than SARS-CoV and MERS-CoV.

The greater infectivity may be associated with the most important holiday in China - the Spring Festival, also known as Chinese New Year or Lunar Year. Similar to the Christmas Day in western countries, Spring Festival is a day for family gathering and reunion, and hundreds of millions of people will return to their hometowns or travel abroad during the week-long holiday. Wuhan as a metropolis in China has an efficient transport system, which promotes the mobility and migration of the population, deteriorating the infection. According to WHO, from May 13, 2020, 213 countries or regions have announced COVID-19 cases, and there have been more than 4.4 million of confirmed cases globally, including nearly 300,000 reported deaths (WHO, 2020c).

With more studies involving genetics, epidemiology, and medicine being conducted, the mystery of COVID-19 has been gradually unveiled. This review aims to consolidate and discuss the likely origins and genetic features of SARS-CoV-2 as well as the recent clinical findings and potential effective treatments of COVID-19.

\section{Origins of the virus}

Coronaviruses are enveloped, positive-sense, single plus - stranded RNA viruses, broadly distributed in humans, mammals, and birds, leading to the respiratory, enteric, and neurologic diseases (Chen et al., 2020). The gamma and delta coronaviruses are from birds, which hardly cause human infections, while the alpha and beta coronaviruses are mainly found in mammals such as bats, rodents, civets, and humans, which are known to cause human diseases (Woo et al., 2005, 2012; Lau et al., 2015). Most human coronavirus infections such as HCoV-229E, HCoV-OC43, HCoV-NL63, HCoV-HKU1 are mild and merely cause the common cold and diarrhoea in humans (Su et al., 2016). The prevalence of the two beta coronaviruses, SARS-CoV and MERS-CoV have been related to sometimes fatal illnesses, with mortality rates of $10 \%$ for SARS-CoV (WHO, 2004) and 37\% for MERS-CoV (WHO, 2020a). It is believed that both SARS-CoV and MERS-CoV originate from bats, with direct transmission to human through market civets and dromedary camels, respectively (Cui et al., 2019). Thanks to extensive research on SARS-CoV and MERS-CoV, SARS-CoV-2 was identified as a SARS-like coronavirus in a short time by epidemiological investigation and genome sequencing (Yang and Chen, 2020; Lu et al., 2020a). Although the intermediate hosts remain to be unclear, it is strongly speculated that the original source of SARS-CoV-2 is the bat, which has been revealed in a very first epidemiological study of a family cluster of COVID-19 (Chan et al., 2020b; Zhang et al., 2020b).

The study recruited a family cluster of six patients who travelled from Shenzhen to Wuhan, and five of them got infected with the SARS-CoV-2. Although the primary cases of COVID-19 were tightly associated with the shared history of exposure to Huanan seafood wholesale market, none of the five patients had a history of contact with wild animals, visiting Huanan seafood wholesale market, or eating game meat. Moreover, another family member who never travelled to Wuhan, became infected with the SARS-CoV-2 after staying several days with four infected family members. Therefore, it is reasonable to assume that COVID-19 is a person-to-person transmitted disease. Based on Chinese authorities, the transmission is mainly via respiratory droplets or close contact. It is transmitted through aerosol by continuous exposure to high concentrations of aerosol in relatively close space. Although SARS-CoV-2 RNA was detected in some stool 
specimens, the capability of transmitting by alimentary tract still requires further investigation.

The study also performed genome and surface spike protein analysis of the PCR amplicon fragments from several infected participants. The full virus genome indicated that SARS-CoV-2 is a beta coronavirus belonging to the lineage or the subgenus Sarbecovirus. Furthermore, the nucleotide identity of the complete genome of SARSCoV-2 and bat-SL-CoVZC45 was $89 \%$, and the nucleotide (nt) identity between the spike protein of SARS-CoV-2 and bat-SL-CoVZC45 was $84 \%$. Moreover, the spike protein of SARS-CoV-2 had a 78\% nt identity with the human SARSCoV. Thus, the genetic evaluations of SARS-CoV-2 have been closest to the bat SARS-related coronaviruses first detected in the Chinese horseshoe bat, Rhinolophus sinicus which inhabits Zhoushan, Zhejiang province, southern part of China, from 2015 to 2017 (Chan et al., 2020b). Afterwards, more and more studies linked to genetics have supported the hypothesis that the transmission chain started from the bat and passed to the human. There is currently no evidence that the bat comes from Wuhan Huanan seafood wholesale market.

\section{Genetics of the virus}

A number of genetic studies have described the genomic characteristics of SARS-CoV-2, using sequencing of bronchoalveolar lavage fluid (BALF) specimens and cultured isolates from different patients (Zhu et al., 2020; Zhou et al., 2020; Wu et al., 2020a). Phylogenetic analysis has suggested that the SARS-CoV-2 is distinct from SARS$\mathrm{CoV}$ and MERS-CoV (Benvenuto et al., 2020). The genetic similarity between the SARS-CoV-2 strains and SARS-CoV is about 79\%, and it is even less between SARS-CoV-2 and MERS-CoV (about 50\%) (Lu et al., 2020). However, it exhibits some genomic and phylogenetic similarity to some beta coronaviruses detected in bats. Thus the SARS-CoV-2 represents a novel virus within the Sabecovirus subgenus, which could be classified into three independent subclades: (1) the SARS-CoV-2 from Wuhan together with the two bat-derived SARS-like strains, bat-SL-CoVZC45 and bat-SL-CoVZXC21 from Zhoushan, Zhejiang, China; (2) SARS-CoV strains from humans and some genetically similar SARS-like coronaviruses from bats detected from southwestern China; (3) two SARS-CoV-related strains from Rhinolophus sp. from Bulgaria and Kenya (Lu et al., 2020; Chan et al., 2020a). Since the SARS-CoV-2 belongs to beta coronaviruses, it has the typical order: a 5 ' untranslated regions (UTR)- replicase complex (orflab)- S gene- $\mathrm{E}$ gene- $\mathrm{M}$ gene- N gene- $3^{\prime}$ UTR, and several unidentified nonstructural open reading frames (ORF) (Zhu et al., 2020). With the more discoveries of SARS-CoV-2 genome being revealed, the more important information on the origins and cell receptor binding of the virus has been provided.

A study has compared SARS-CoV-2 with SARS-CoV and bat SARS-like coronavirus, using fast unconstrained Bayesian approximation (FUBAR) analysis to deduce site-specific pervasive selection and episodic diversifying selection across interested regions, and to identify episodic selection at each site (Murrell et al., 2012, 2013; Benvenuto et al., 2020).

On the $S$ region, two individual sites $\left(536^{\text {th }}\right.$ and $644^{\text {th }}$ nt position) showed a significant $(\mathrm{P}<0.05)$ pervasive episodic selection. Concerning the SARS-CoV-2 present in the same clade with bat-derived SARS-like coronavirus, an asparagine residue replaces an aspartic residue on the $536^{\text {th }}$ aa position, and a threonine residue replaces an alanine residue on the $644^{\text {th }}$ aa position. Additionally, FUBAR analysis has indicated a significant $(\mathrm{P}<0.05)$ pervasive negative selection in 1065 sites (87\%), implying high conservation in the $S$ region (Benvenuto et al., 2020).

Two sites $\left(380^{\text {th }}\right.$ and $410^{\text {th }}$ nt position) had significant $(\mathrm{P}<0.05)$ pervasive episodic selection on the $\mathrm{N}$ region. $\mathrm{Re}-$ garding the SARS-CoV-2 in the same clade with bat-derived SARS-like coronavirus, a glutamine residue replaces an asparagine residue on the $380^{\text {th }}$ aa position, and a threonine residue replaces an alanine residue on the $410^{\text {th }}$ aa position. Besides, FUBAR analysis has shown a significant $(\mathrm{P}<0.05)$ pervasive negative selection in six sites $(14 \%)$. However, no sites on the $\mathrm{E}$ and $\mathrm{M}$ regions exhibited positive selection (Benvenuto et al., 2020).

From the structural and molecular analysis of the nucleocapsid of SARS-CoV-2, SARS-CoV-2 and bat-derived SARS-like coronavirus have common aminoacidic sequences on the $309^{\text {th }}$ and $380^{\text {th }}$ aa positions. While SARSCoV-2 differs from SARS-CoV in these two positions. Specifically, SARS-CoV-2 has a polar amino acid, but SARS-CoV has a nonpolar aa at these positions (position 309 or 380 or both) (Benvenuto et al., 2020).

The further selective pressure and structural analysis has demonstrated the stability of the viral particle of SARS-CoV-2 resulting from mutation of surface protein (spike protein) and nucleocapsid N protein. The viral spike protein plays an important role in virus entry into cell and the $\mathrm{N}$ protein is responsible for virus transcription and assembly efficiency, which are closely associated with infection and pathogenesis of the virus. Therefore, the mutation of these proteins confers two pivotal features of SARS-CoV-2: (1) a stronger infection and pathogenicity than the bat-like SARS coronavirus; (2) a weaker pathogenicity compared to SARS-CoV (Benvenuto et al., 2020).

To better understand the potential of SARS-CoV-2 to infect humans, studies compared the receptor-binding domain (RBD) of several coronaviruses. There were relatively high similarities of amino acid sequences and predicted 
protein structures between SARS-CoV-2 RBD and SARSCoV RBD (73.8\%-74.9\% aa identity) (Lu et al., 2020), which suggested that SARS-CoV-2 is able to use the human ACE2 receptor to enter the cell (Hu et al., 2017; Li et al., 2020b; Hoffmann et al., 2020), despite mutations of spike protein. Thus, human to human transmission is strengthened.

Furthermore, a study collected BALF and human airway epithelial cell virus isolates from three patients and performed Illumina and nanopore sequencing. The study has demonstrated that the genome of SARS-CoV-2 has an $86.9 \%$ nt sequence identity to bat-SL-CoVZC45 genome (Zhu et al., 2020). Another study with a similar result showed a nucleotide identity of $89.1 \%$ between SARS-CoV-2 and bat-SL-CoVZC45. Meanwhile, this study has suggested that the predicted whole genome sequences of SARS-CoV-2 has $29903 \mathrm{nt}$. The 5 ' terminal sequences of SARS-CoV- 2 are $265 \mathrm{nt}$, and the 3 ' terminal sequences are $229 \mathrm{nt}$ long. The predicted length of replicase complex (orflab) is $20291 \mathrm{nt}$, containing 16 predicted nonstructural proteins, with at least 13 ORFs downstream. Moreover, the predicted length of S, E, M, N gene is 3822, 228, 669 and $1260 \mathrm{nt}$, respectively. Additionally, the SARS-CoV-2 contains consistent ORFs regions with other members of the subgenus Sarbecovirus. Also, it carries a predicted ORF8 gene (366 nt long) located between the M and N ORF genes (Wu et al., 2020a).

A recent study compared full-length sequencing between SARS-CoV-2 and bat CoV RaTG13 that was previously found in Rhinolophus affinis from Yunan province. A high similarity through the genome was detected by simplot analysis, with $96.2 \%$ overall identity. Moreover, the phylogenetic analysis of full-length genome, RNAdependent RNA polymerase ( $\mathrm{RdRp}$ ) gene, and $\mathrm{S}$ gene sequences all suggested that RaTG13 has the closest association with SARS-CoV-2, which forms an independent lineage. Furthermore, the sequence identity of SARS-CoV-2 S gene is significantly higher in RaTG13 (93.1\% nt identity) than in other bat-derived SARS-like coronaviruses (less than $75 \%$ nt identity). In addition, no evidence of recombination events was found in the genome of SARS-CoV-2, in comparison to the aligned genome sequences of RaTG13, SARS-CoV and several bat-derived SARS-like coronaviruses (Zhou et al., 2020).

As mentioned above, SARS-CoV-2 is homologous and genetically similar to several bat SARS like coronaviruses, supporting the hypothesis that the transmission chain begun in the bat and then infected humans. However, despite the importance of bat, there is some evidence that another animal plays a role as an intermediate host between bat and human (Zhang et al., 2020b). In fact, most bat species are hibernating when the COVID-19 outbroke in December in Wuhan. Moreover, no bats were under trade at Huanan seafood wholesale market. Besides, no direct ancestors of SARS-CoV-2 were confirmed yet. Finally, there are other animals acting as intermediate hosts between bat and human in SARS-CoV (masked palm civet) and MERS-CoV (dromedary camel) (Guan et al., 2003; Alagaili et al., 2014; Lu et al., 2020). Therefore, it seems likely that bat acts as the natural reservoir of SARS-CoV-2 which has been transmitted to humans by currently unknown animals traded at Huanan seafood wholesale market.

\section{Clinical findings in the patients}

Extensive research on COVID-19 has driven the discovery of the epidemiological, clinical, laboratory, and radiological characteristics of infected people. Two studies conducted in Wuhan enrolled 41 and 99 patients, to figure out the manifestations of the disease. In the first study (Huang et al., 2020), 41 patients were identified as laboratory-confirmed COVID-19 positive and admitted to a designated hospital in Wuhan. After reviewing clinical charts, nursing records, laboratory findings, and chest $\mathrm{x}$-rays of these patients, the study has demonstrated that $73 \%$ of infected patients were men, and more than $30 \%$ of patients had underlying health problems, involving diabetes, hypertension, and cardiovascular disease. The median age of all participants was 49 years. Additionally, $66 \%$ of patients had a history of exposure to Huanan seafood wholesale market (Huang et al., 2020).

COVID-19 may be asymptomatic and transmissible to another patient at the same time (Al-Tawfiq, 2020; Chen, 2020). Also, the disease may reactivate (Chen et al., 2020a; Ye et al., 2020). However, the most common symptoms at onset of the disease were fever, which accounted for $98 \%$ of initial symptoms (Huang et al., 2020). Cough, myalgia or fatigue, and sputum production took up to $76 \%, 44 \%$, and $28 \%$ of initial symptoms, respectively. Less common symptoms at the beginning of disease were headache, haemoptysis, and diarrhoea. Besides, dyspnoea occurred in more than half of participants; almost $30 \%$ of patients developed acute respiratory distress syndrome (ARDS); more than $30 \%$ of patients were admitted to an ICU. Other complications include RNAemia (15\%), acute cardiac injury (12\%), and secondary infection (10\%). Furthermore, the median duration from initial symptoms to initial hospital admission was 7 days, to breathlessness was 8 days, to ARDS was 9 days, to mechanical ventilation was 1.5 days and to ICU admission was 10.5 days.

The blood specimens of participants showed that $63 \%$ of patients had lymphopenia (lymphocyte count less than $1.0 \times 10^{9}$ ) and $25 \%$ of patients had leucopenia (white blood cell count less than $4.0 \times 10^{9}$ ) (Huang et al., 2020). In comparison to ICU patients, the median prothrombin time and the median D-dimer level were significantly lower in non- 
ICU patients. Levels of aspartate aminotransferase and hypersensitive troponin were increased in $37 \%$ and $12 \%$ of total patients respectively. Those who had increased levels of hypersensitive troponin all developed virusrelated cardiac injury. Moreover, four patients were accompanied with secondary infections after admitting to ICU, 3 of them had elevated levels of procalcitonin which was assessed as normal in $69 \%$ of patients. Furthermore, there was no difference in plasma levels of IL5, IL12p70, IL15, Eotaxin, and RANTES between COVID-19 and healthy people. However, initial plasma IL1B, IL1RA, IL7, IL8, IL9, IL10, basic FGF, GCSF, GMCSF, IFN $\gamma$, IP10, MCP1, MIP1A, MIP1B, PDGF, TNFa, and VEGF concentrations increased in all patients. Besides, the plasma levels of IL2, IL7, IL10, GCSF, IP10, MCP1, MIP1A, and TNFa increased in ICU patients in comparison to non-ICU patients.

All 41 patients had abnormal chest CT images, among which, 40 had bilateral degeneration in lungs (Huang et al., 2020). The typical findings of bilateral ground-glass opacity and subsegmental areas of consolidation, with the development of the disease, the consolidation resolved, and only bilateral ground-glass opacity remained (Huang et al., 2020; Xu et al., 2020b). Whereas, the representative findings of chest CT images of ICU patients were bilateral multiple lobular and subsegmental areas of consolidation.

In the second study (Chen et al., 2020b), the epidemiological findings have been consistent with the first study, middle-aged men comprise the major group of patients and 47 of 99 patients were present at Huanan seafood wholesale market for a long time. More than half of patients had underlying diseases which were more multiple than in the first study, including cardiovascular and cerebrovascular, endocrine, digestive, respiratory, nervous diseases, and malignant tumour. In addition, the clinical presentations have been in accordance with the first study, patients suffered from fever, cough, shortness of breath, headache, myalgia, confusion, chest pain, and diarrhoea. Other complications of the second study were acute respiratory injury, acute renal injury, septic shock, and ventilator associated pneumonia.

Among total 99 patients, lymphocytes and haemoglobin had lower levels than in healthy people, while the white blood cell count varied, where 9 patients were under the normal range and 24 patients were above the normal range (Chen et al., 2020b). Especially neutrophils were above the normal range in 38 patients. Meanwhile, there were high or low levels of platelets in different participants. Moreover, 43 patients had different degrees of liver function damage, with changed levels of alanine aminotransferase or aspartate aminotransferase. Furthermore, 7 patients had different degrees of renal function abnormality with the increased levels of blood urea nitrogen or serum creatinine. None of patients were infected by another respiratory virus. One case of bacterial infection was diagnosed as Acinetobacter baumannii, Klebsiella pneumoniae, and Aspergillus flavus. Candida glabrata was positive in one patient, and Candida albicans was positive in three patients. Besides, the findings of chest CT images were in line with the first study.

Despite the increasing number of confirmed cases, the clinical investigation of patients was previously limited to Wuhan, therefore, more studies need to be done outside Wuhan in order to fully understand the characteristics of COVID-19. So far, a clinical study in Zhejiang province and a case report in the USA can provide more information of COVID-19. The 62 participants from Zhejiang in the clinical study were never exposed to the Huanan seafood wholesale market. Their symptoms were similar to the above mentioned two studies, whereas, the number of patients who developed severe complications was far less than two studies in Wuhan. Moreover, only one patient was admitted to an ICU. Therefore, compared to initial patients in Wuhan, the symptoms of patients in Zhejiang are more mild (Xu et al., 2020a).

The initial clinical presentations in American case were mild cough and low-grade intermittent fevers, with progression to pneumonia on day 9 of the patient's illness, of which the timing is consistent with later onset of dyspnoea assessed in the first study. Noteworthily, SARS-CoV-2 RNA was detected in a stool specimen collected on day 7 of the patient's illness, but the clinical significance needs further investigation (Holshue et al., 2020).

Recently, a study has first reported the pathological characteristics of a patient who died on day 13 of illness because of severe infection after diagnosing COVID-19 by obtaining biopsy samples at autopsy. The biopsy samples were taken from lungs, liver, and heart tissue. The pathological changes were bilateral diffuse alveolar injury with cellular fibromyxoid exudation indicated in histological examination. Moreover, early-stage ARDS was found in the left lung with pulmonary edema and hyaline membrane formation. ARDS was found in the right lung with significant debonding of pneumocytes and hyaline membrane formation. In addition, interstitial mononuclear inflammatory infiltrates especially lymphocytes were detected bilaterally. Furthermore, viral cytopathic like changes were shown in the intra-alveolar spaces that multinucleated syncytial cells with atypical hypertrophied pneumocytes represented as large nuclei, amphophilic granular cytoplasm and dominant nucleoli. Besides, the biopsy specimens of liver showed moderate microvascular steatosis and mild lobular and portal activity, suggesting that the potential reason could be COVID-19 or drug-induced liver damage. What's more, no substantial injury found in the heart tissue except for mild interstitial mononuclear inflammation, indicating 
COVID-19 is directly associated with heart impairment. In addition, the flow cytometric analysis of the patient's peripheral blood implied that overactivation of $\mathrm{T}$ cells, presented by elevated levels of Th17 and high cytotoxicity of CD8 T cells, partially contributes to impaired immune system (Xu et al., 2020c).

According to multiple studies including one mentioned above, Chinese authorities have reported that the latent period of COVID-19 is 1 to 14 days, dominated by 3 to 7 days. However, CCTV news reported two confirmed cases with particularly long latent periods which were 34 and 94 days in Xinyang, Henan province. Additionally, positive realtime RT-PCR or genetic sequencing is considered as gold standard to diagnose SARS CoV-2. COVID-19 according to symptoms has been classified to four types. Light type is defined as mild clinical symptoms such as fever, cough, or shortness of breath, without changes of chest CT image. Normal type is identified as typical clinical symptoms accompanied with abnormalities in chest CT image. Severe type is diagnosed if patients present respiratory distress ( $R R>30 / \mathrm{min}$ ), or finger oxygen saturation $\leq 93 \%$ at rest, or arterial partial pressure of oxygen / fraction of inspired oxygen $\left(\mathrm{PaO}_{2} / \mathrm{FiO}_{2}\right) \leq 300 \mathrm{mmHg}$. Patients with light or normal type with evident progression (>50\%) in 24 to 48 hours based on chest CT image should be treated as severe type. Critical type is diagnosed if patients have at least one of the following symptoms: respiratory failure with mechanical ventilation, shock or was admitted to an ICU because of other organ failures. It is notable that appropriate quarantine is necessary for discharged patients too, since several Chinese people had recrudescence a few days after negative real-time RT-PCR (National Heath Commission of the People's Republic of China, 2020).

\section{Current and potential treatments}

According to previous clinical studies and Chinese National Health Commission (NHC) (Zhang and Liu, 2020; Zu et al., 2020; Wu and McGoogan, 2020), the current treatments include oxygen therapy, antiviral therapy, empirical antibiotic therapy, systematic corticosteroids, convalescent plasma therapy, traditional Chinese medicine (herbal medicine and moxibustion), and potential interventions are anti-viral drugs and vaccination. The state of illness determines which treatment should be given. The general interventions involving sufficient rest, ample nutrition, balance of water and electrolytes, monitor of vital signs and finger oxygen saturation, and etc. are beneficial to every patient. Moreover, it is necessary to do laboratory examinations such as routine tests of blood, urine, stool, CRP, biochemical indexes of liver, heart, kidneys, chest CT, and cytokine detections if condi- tional, in order to predict prognosis and select appropriate therapies. Besides, oxygen therapy should be provided as soon as possible, based on patients' situation, nasal catheter, mask, and transnasal high flow oxygen therapy served as potential choices.

Currently, there are no approved antiviral drugs for COVID-19 at present. Although in the case report from the USA that the patient's clinical condition improved in one day after injecting remdesivir (a novel nucleotide analogue prodrug) into vein with no adverse events. The potent efficacy to treat COVID-19 is still under investigation with several experiments and clinical trials that have been carried out in China and other countries (Robson, 2020; Sheahan et al., 2020; Elfiky,2020). Additionally, other broad-spectrum antiviral drugs mentioned in several clinical studies involving oseltamivir, lopinavir, ritonavir, and ganciclovir have been all applied to treat COVID-19 based on the experience of SARS and MERS. Chinese NHC has suggested some antiviral medications involving alpha interferon (twice daily, aerosol inhalation), lopinavir/ritonavir (400 mg/100mg, twice daily, orally), chloroquine phosphate (500 mg, based on body weight, once or twice daily, orally), arbidol (200 mg, three times a day, orally), and combination of ribavirin ( $500 \mathrm{mg}$, twice or three times a day, intravenously) and alpha interferon or lopinavir/ ritonavir (Deng et al., 2020; Devaux et al., 2020; Gautret et al., 2020). It is notable that except for alpha interferon, the application of other antiviral drugs should be no more than 10 days. It is also not recommended to use three or more drugs simultaneously. Moreover, neither adverse effects are supposed to be underestimated, nor interplay among various drugs should be ignored.

Antibiotic therapy can also be administered to COVID-19 patients according to the severity of concurrent bacterial infection. The antibiotics include cephalosporins, quinolones, carbapenems, tigecycline against methicillin-resistant Staphylococcus aureus, linezolid, and antifungal drugs. Systematic corticosteroid intervention is served as a combined regimen if there are high amounts of cytokines induced by COVID-19, which can reduce inflammatory-induced lung damage. However, previous evidence of SARS and MERS have indicated that receiving large amounts of corticosteroids is associated with delayed virus clearance, due to immunosuppression. Therefore, corticosteroids should not be applied routinely, and the recommended dosage of glucocorticoids is supposed to be lower than the relative dosage of methylprednisolone ( $1 \sim 2 \mathrm{mg} / \mathrm{kg} / \mathrm{day})$ according to Chinese guideline.

Severe and critical types of patients should not only be given the interventions mentioned above, but to prevent complications, secondary infections, and development of chronic underlying disease. Thus, nasal catheter or mask 
oxygen support is vital to alleviate respiratory distress and hypoxaemia of patients. If the situations of respiratory distress and hypoxaemia remains unsolved, transnasal high flow oxygen therapy or noninvasive ventilator mechanical ventilation should be used as soon as possible. Invasive ventilator to assist ventilation is supposed to be applied when after using transnasal high flow oxygen therapy or noninvasive ventilator mechanical ventilation in a short time ( 1 to 2 hours) is non-effective. The protective strategy of invasive ventilator assisted breathing therapy is adjustment of low tidal volume ( 6 to $8 \mathrm{ml} / \mathrm{kg}$ ideal body weight) and low inspiratory pressure (plateau pressure less than $30 \mathrm{~cm} \mathrm{H}_{2} \mathrm{O}$ ). Moreover, sedation and muscle relaxant are important to reduce asynchronism between patient and ventilatory machine. Besides, for patients with severe ARDS, it is recommended to apply lung recruitment maneuver or extracorporeal membrane oxygenation (ECMO) to improve ventilation function.

In addition, it is believed that sufficient fluid is the guarantee to improve microcirculation, and vasoactive drug is able to be used if necessary. Convalescent plasma therapy is suitable for severe or critical types of patients, and patients with rapid progression of COVID-19. According to Chinese authorities, the initial eleven patients who received convalescent plasma therapy in Wuhan, had an extreme improvement (Zhang et al., 2020a). Also, six of them were infected by both bacteria and virus which disappeared in two or three days after treating with convalescent plasma. Moreover, for children with severe infection, r-globulin should be considered to apply intravenously based on the experience.

Traditional Chinese Medicine (TCM) may also be useful to tackle COVID-19 (Liu, 2020; ul Qamar et al., 2020). In accordance with the basic theories of TCM, heat-clearing and detoxifying herbs should be mainly used to treat COVID-19. Therefore, it is recommended to use Jinhua Qinggan granules, Lianhua Qingwen granules, or Huoxiang Zhengqi liquid in order to mitigate fatigue, fever, and gastrointestinal problems during medical observation period (Runfeng et al., 2020). For confirmed cases, lungclearing and detoxifying herbs such as Ephedra, Gypsum, Almond, Scutellaria, Bupleurum, and Forsythia are beneficial to alleviate symptoms like fever, cough, myalgia, sputum production, headache, haemoptysis, diarrhea, and dyspnea. Data from the State Administration of Traditional Medicine of China that in 701 confirmed cases who received herbal medicine implied potent efficacy. 130 patients were discharged, symptoms of 51 patients disappeared, 268 cases had significant improvement, and 212 cases' conditions were stable without deteriorating. Additionally, the application of moxibustion is associated with improvement of immunity. ST36 (Zusanli), LI4 (Hegu), LR3 (Taichong), RN8 (Shenque) are common treatments to apply. However, more study still needs to be done in order to understand if certain herbal medicines can indeed treat COVID-19.

Although worldwide scientists are making full use of every minute to develop the vaccine, it still needs relatively long time to successfully administer vaccine to human beings. The latest research of Westlake University has reported that the antibodies were produced by initial vaccine, however animal experiments needs to be conducted subsequently.

\section{Conclusion and perspectives}

In conclusion, the outbreak of COVID-19 extremely jeopardizes the global public health. Although the initial case was found at Huanan seafood wholesale market in Wuhan, whether the SARS-CoV-2 originates from Wuhan is still a mystery. However, there is one certain thing that the virus comes from bats, with $96.2 \%$ overall identity of bat CoV RaTG13. The most common symptoms of COVID-19 are fever, cough, and fatigue, some people may show shortness of breath, headache, myalgia, and diarrhoea. With the progression of the disease, ARDS, acute cardiac injury, secondary infection or other complications may occur. Unfortunately, there is currently no specific antiviral medicine for COVID-19, even the potentially potent drug remdesivir is still on clinical trial. At present, symptomatic treatments are the major interventions. Sufficient rest, ample nutrition, balance of water and electrolytes, oxygen therapy and Chinese medicine have played vital roles in recovery of patients in China.

Although the confirmed cases reported in China decreased significantly over past few days, the unreported cases are robustly associated with the management of the disease. Considering that some of the unreported cases are not isolated and patients having no idea that they are infected, large cities apart from Wuhan or overseas with close transport links to China could become outbreak epicenters. Indeed, more and more confirmed cases have been reported in countries like Japan, Korea, Italy and America. As outbreaks take place globally, the management of critically ill patients with COVID-19 infection will be challenging. Therefore, substantial public health interventions at both the population and individual levels are supposed to be implemented immediately. Preparedness plans and mitigation interventions including securing supply chains of pharmaceuticals, hospital resources, personal protective tools and necessities should be carried out worldwide as soon as possible.

Furthermore, there are still many unsolved problems: (1) Where is the exact origin of virus? Whether origin of the virus was in Wuhan? The outbreak in Wuhan needs 
further investigation. (2) It is unlikely that SARS-CoV-2 directly infects humans from bats, so identifying the presently unknown intermediate host is beneficial for the management of COVID-19. (3) Psychological health is important for both infected patients and healthy people, however, only a few organizations mainly at schools and hospitals provide psychological counselling for insiders. (4) When will the virus disappear, or will it continue to exist with relatively low infection and pathogenicity like flu? Effective vaccine is necessary to prevent the second or third outbreak of the infection.

\section{References}

Al-Tawfiq JA., Travel Med. Infect. Dis. 101608, 2020. https://doi. org/10.1016/j.tmaid.2020.101608

Alagaili AN, Briese T, Mishra N, Kapoor V, Sameroff SC, Burbelo PD, de Wit E, Munster VJ, Hensley LE, Zalmout IS, Kapoor A, Epstein JH, Karesh WB, Daszak P, Mohammed OB, Lipkin WI., mBio 5 e00884, 2014. https://doi.org/10.1128/mBio.01002-14

Bauch CT, Lloyd-Smith JO, Coffee MP, Galvani AP., Epidemiology (Cambridge, Mass.) 16, 791-801, 2005. https://doi.org/10.1097/01.ede.0000181633.80269.4C

Benvenuto D, Giovanetti M, Ciccozzi A, Spoto S, Angeletti S, Ciccozzi M., J. Med. Virol. 92, 455-459, 2020. https://doi.org/10.1002/jmv.25688

Centre for Health Protection of the Hong Kong Special Administrative Region Government., CHP provides further information on cluster of pneumonia cases in Wuhan. Jan 12, 2020, 2020. https://www.info.gov.hk/gia/general/202001/12/P2020011200710.htm

Chan JF-W, Kok K-H, Zhu Z, Chu H, To KK-W, Yuan S, Yuen K-Y., Emerg. Microbes Infect. 9, 221-236, 2020a. https://doi. org/10.1080/22221751.2020.1719902

Chan JF-W, Yuan S, Kok K-H, To KK-W, Chu H, Yang J, Xing F, Liu J, Yip CC-Y, Poon RW-S, Tsoi H-W, Lo SK-F, Chan K-H, Poon VK-M,Chan W-M, Ip JD, Cai J-P, ChengVC-C, Chen H, Hui CK-M, Yuen K-Y., Lancet (London) 395, 514-523, 2020b. https://doi.org/10.1016/S0140-6736(20)30154-9

Chen D, Xu W, Lei Z, Huang Z, Liu J, Gao Z, Peng L, Int. J. Infect. Dis. 93, 297-299, 2020a. https://doi.org/10.1016/j. ijid.2020.03.003

Chen J., Microbes Infect, 22, 69-71, 2020. https://doi.org/10.1016/j. micinf.2020.01.004

Chen N, Zhou M, Dong X, Qu J, Gong F, Han Y, Qiu Y, Wang J, Liu Y, Wei Y, Xia Ja, Yu T, Zhang X, Zhang L., Lancet (London) 395, 507-513, 2020b. https://doi.org/10.1016/ $\underline{\text { S0140-6736(20)30211-7 }}$

Chen Y, Liu Q, Guo D., J. Med. Virol. 92, 418-423, 2020c. https:// doi.org/10.1002/jmv.25681

Cohen J, Normile D., Science (New York) 367, 234-235, 2020. https://doi.org/10.1126/science.367.6475.234

Cui J, Li F, Shi Z-L., Nature reviews. Microbiology 17,181-192, 2019. https://doi.org/10.1038/s41579-018-0118-9
Deng L, Li C, Zeng Q, Liu X, Li X, Zhang H, Hong Z, Xia J., J. Infect, 81, e1-e5, 2020. https://doi.org/10.1016/j. jinf.2020.03.002

Devaux CA, Rolain J-M, Colson P, Raoult D., Int. J. Antimicrob. Agents, 105938, 2020. https://doi.org/10.1016/i.ijantimicag.2020.105938

Elfiky AA., Life Sciences, 117592, 2020. https://doi.org/10.1016/j. lfs.2020.117592

Gautret P, Lagier J-C, Parola P, Meddeb L, Mailhe M, Doudier B, Courjon J, Giordanengo V, Vieira VE, Dupont HT., Int. J. Antimicrob. Agents, 105949, 2020. https://doi. org/10.1016/j.ijantimicag.2020.105949

Guan Y, Zheng BJ, He YQ, Liu XL, Zhuang ZX, Cheung CL, Luo SW, Li PH, Zhang LJ, Guan YJ, Butt KM, Wong KL, Chan KW, Lim W, Shortridge KF, Yuen KY, Peiris JSM, Poon LLM., Science (New York) 302, 276-278, 2003.

Hoffmann M, Kleine-Weber H, Schroeder S, Krüger N, Herrler T, Erichsen S, Schiergens TS, Herrler G, Wu N-H, Nitsche A., Cell, 181, 271-280, 2020. https://doi.org/10.1016/j. cell.2020.02.052

Holshue ML, DeBolt C, Lindquist S, Lofy KH, Wiesman J, Bruce H, Spitters C, Ericson K, Wilkerson S, Tural A, Diaz G, Cohn A, Fox L, Patel A, Gerber SI, Kim L, Tong S, Lu X, Lindstrom S, Pallansch MA, Weldon WC, Biggs HM, Uyeki TM, Pillai SK, Washington State -nCo VCIT., N. Engl. J. Med. 10.1056/NEJMoa2001191, 2020.

Hu B, Zeng L-P, Yang X-L, Ge X-Y, Zhang W, Li B, Xie J-Z, Shen X-R, Zhang Y-Z, Wang N, Luo D-S, Zheng X-S, Wang M-N, Daszak P, Wang L-F, Cui J, Shi Z-L., PLoS Pathog. 13 e1006698-e1006698, 2017. https://doi.org/10.1371/ journal.ppat.1006698

Huang C, Wang Y, Li X, Ren L, Zhao J, Hu Y, Zhang L, Fan G, Xu J, Gu X, Cheng Z, Yu T, Xia J, Wei Y, Wu W, Xie X, Yin W, Li H, Liu M, Xiao Y, Gao H, Guo L, Xie J, Wang G, Jiang R, Gao Z, Jin Q, Wang J, Cao B., Lancet (London) 395, 497-506. 2020. https://doi.org/10.1016/S0140-6736(20)30183-5

Ksiazek T., N. Engl. J. Med. 348, 2003.

Lau S, Woo P, Li K, Tsang A, Fan R, Luk H, Cai J, Chan K, Zheng B, Wang M, J. Virol. 89, 3076-3092, 2015. https://doi. org/10.1128/JVI.02420-14

Li Q, Guan X, Wu P, Wang X, Zhou L, Tong Y, Ren R, Leung KSM, Lau EHY, Wong JY, Xing X, Xiang N, Wu Y, Li C, Chen Q, Li D, Liu T, Zhao J, Li M, Tu W, Chen C, Jin L, Yang R, Wang Q, Zhou S, Wang R, Liu H, Luo Y, Liu Y, Shao G, Li H, Tao Z, Yang Y, Deng Z, Liu B, Ma Z, Zhang Y, Shi G, Lam TTY, Wu JTK, Gao GF, Cowling BJ, Yang B, Leung GM, Feng Z., N. Engl. J. Med. 10.1056/NEJMoa2001316, 2020a.

Li X, Geng M, Peng Y, Meng L, Lu S., J. Pharm. Anal., 10, 102-108, 2020b. https://doi.org/10.1016/j.jpha.2020.03.001

Liu C-X., Chin. Herb. Med., 12, 97-103, 2020. https://doi. org/10.1016/j.chmed.2020.03.004

Liu Y, Gayle AA, Wilder-Smith A, Rocklöv J., J. Travel Med. 27, 2020. https://doi.org/10.1093/jtm/taaa021

Lu R, Zhao X, Li J, Niu P, Yang B, Wu H, Wang W, Song H, Huang B, Zhu N, Bi Y, Ma X, Zhan F, Wang L, Hu T, Zhou H, Hu Z, Zhou W, Zhao L, Chen J, Meng Y, Wang J, Lin Y, Yuan J, Xie Z, Ma J, Liu WJ, Wang D, Xu W, Holmes EC, 
Gao GF, Wu G, Chen W, Shi W, Tan W., Lancet (London) 395, 565-574. 2020. https://doi.org/10.1016/S01406736(20)30251-8

Murrell B, Moola S, Mabona A, Weighill T, Sheward D, Kosakovsky Pond SL, Scheffler K., Mol. Biol. Evol.30,1196-1205, 2013. https://doi.org/10.1093/molbev/mst030

Murrell B, Wertheim JO, Moola S, Weighill T, Scheffler K, Kosakovsky Pond SL., PLoS Genet. 8, e1002764-e1002764, 2012. https://doi.org/10.1371/journal.pgen.1002764

National Health Commission of the People's Republic of China., Diagnosis and Treatment of COVID-19, http://www. nhc.gov.cn/yzygj/s7653p/202002/8334a8326dd94d 329df351d7da8aefc2/files/b218cfeb1bc54639af227f922bf6b817.pdf, 2020.

Robson B., Comput. Biol. Med. 119, 103670, 2020. https://doi. org/10.1016/j.compbiomed.2020.103670

Romulus, Breban, Julien, Riou, Arnaud, Fontanet., Lancet (London) 382, 694-699, 2013. https://doi.org/10.1016/ S0140-6736(13)61492-0

Runfeng L, Yunlong H, Jicheng H, Weiqi P, Qinhai M, Yongxia S, Chufang L, Jin Z, Zhenhua J, Haiming J., Pharmacol. Res. 382, 694-699, 2020.

Sheahan TP, Sims AC, Leist SR, Schäfer A, Won J, Brown AJ, Montgomery SA, Hogg A, Babusis D, Clarke MO, Spahn JE, Bauer L, Sellers S, Porter D, Feng JY, Cihlar T, Jordan R, Denison MR, Baric RS., Nat. Commun. 11, 222-222. 2020. https://doi.org/10.1038/s41467-019-13940-6

Su S, Wong G, Shi W, Liu J, Lai ACK, Zhou J, Liu W, Bi Y, Gao GF., Trends Microbiol. S0966842X16000718. 2016.

Qamar MTU, Alqahtani SM, Alamri MA, Chen L-L., J. Pharm. Anal. 10, 313-319, 2020. https://doi.org/10.1016/j.jpha. 2020.03.009

WHO, WHO Director-General's remarks at the media briefing on 2019-nCoV on 11 February, https://www.who.int/dg/ speeches/detail/who-director-general-s-remarks-atthe-media-briefing-on-2019-ncov-on-11-february-2020, 2020a.

WHO, Middle East respiratory syndrome coronavirus (MERSCoV), https://www.who.int/emergencies/mers-cov/ en/ 2020b.

WHO, Novel Coronavirus (2019-nCoV). Situation report-5. 25 January 2020., https://www.who.int/docs/defaultsource/coronaviruse/situation-reports/20200125sitrep-5-2019-ncov.pdf?sfvrsn=429b143d_8, 2020c.

WHO, Summary of probable SARS cases with onset of illness from 1 November 2002 to 31 July 2003. Dec 31, 2003., https://www.who.int/csr/sars/country/table2004_04_21/ en/,2004d.

Woo PCY, Lau SKP, Chu C-m, Chan K-h, Tsoi H-w, Huang Y, Wong BHL, Poon RWS, Cai JJ, Luk W-K., J. Virol. 79, 884-895, 2005. https://doi.org/10.1128/JVI.79.2.884-895.2005
Woo PCY, Lau SKP, Lam CSF, Lau CCY, Tsang AKL, Lau JHN, Bai R, Teng JLL, Tsang CCC, Wang M, Zheng B-J, Chan K-H, Yuen K-Y., J. Virol. 86, 3995-4008, 2012. https://doi. org/10.1128/JVI.06540-11

Wu F, Zhao S, Yu B, Chen Y-M, Wang W, Song Z-G, Hu Y, Tao Z-W, Tian J-H, Pei Y-Y, Yuan M-L, Zhang Y-L, Dai F-H, Liu Y, Wang Q-M, Zheng J-J, Xu L, Holmes EC, Zhang Y-Z. Nature 10.1038/s41586-41020-42008-41583, 2020.

Wu JT, Leung K, Leung GM., Lancet (London), S0140-6736(0120), 30260-30269. 2020.

Wu Z, McGoogan JM., JAMA, 10.1001/jama.2020.2648, 2020.

Xu X-W, Wu X-X, Jiang X-G, Xu K-J, Ying L-J, Ma C-L, Li S-B, Wang H-Y, Zhang S, Gao H-N, Sheng J-F, Cai H-L, Qiu Y-Q, Li L-J., BMJ (Clinical Research Ed.) 368, m606-m606. 2020a. https://doi.org/10.1136/bmi.m606

Xu Y-H, Dong J-H, An W-M, Lv X-Y, Yin X-P, Zhang J-Z, Dong L, Ma X, Zhang H-J, Gao B-L., J. Infect. 8, 394-400, 2020b. https://doi.org/10.1016/j.jinf.2020.02.017

Xu Z, Shi L, Wang Y, Zhang J, Huang L, Zhang C, Liu S, Zhao P, Liu H, Zhu L, Tai Y, Bai C, Gao T, Song J, Xia P, Dong J, Zhao J, Wang F-S., Lancet Respir. Med. S2213-2600(2220)30076X. 2020c.

Yang C-W, Chen M-F., J. Microbiol. Immunol. 53, 419-424, 2020. https://doi.org/10.1201/9780429445552-53

Ye G, Pan Z, Pan Y, Deng Q, Chen L, Li J, Li Y, Wang X., J. Infect. 2020.

Zaki AM, van Boheemen S, Bestebroer TM, Osterhaus ADME, Fouchier RAM., N. Engl. J. Med. 367, 1814-1820. 2012. https://doi.org/10.1056/NEJMoa1211721

Zhang B, Liu S, Tan T, Huang W, Dong Y, Chen L, Chen Q, Zhang L, Zhong Q, Zhang X., Chest. S0012-3692:30571-7,2020a.

Zhang L, Liu Y., J. Med. Virol.10.1002/jmv.25707. 2020.

Zhang T, Wu Q, Zhang Z., Current Biol. 30, 1346-1351, 2020b. https://doi.org/10.1016/j.cub.2020.03.022

Zhong NS, Zheng BJ, Li YM, Poon, Xie ZH, Chan KH, Li PH, Tan SY, Chang Q, Xie JP, Liu XQ, Xu J, Li DX, Yuen KY, Peiris, Guan Y., Lancet (London) 362, 1353-1358, 2003. https://doi.org/10.1016/S0140-6736(03)14630-2

Zhou P, Yang X-L, Wang X-G, Hu B, Zhang L, Zhang W, Si H-R, Zhu Y, Li B, Huang C-L, Chen H-D, Chen J, Luo Y, Guo H, Jiang R-D, Liu M-Q, Chen Y, Shen X-R, Wang X, Zheng X-S, Zhao K, Chen Q-J, Deng F, Liu L-L, Yan B, Zhan F-X, Wang Y-Y, Xiao G-F, Shi Z-L., Nature 10.1038/s4158641020-42012-41587, 2020.

Zhu N, Zhang D, Wang W, Li X, Yang B, Song J, Zhao X, Huang B, Shi W, Lu R, Niu P, Zhan F, Ma X, Wang D, Xu W, Wu G, Gao GF, Tan W, China Novel Coronavirus I, Research T., N. Engl. J. Med. 382, 727-733, 2020. https://doi. org/10.1056/NEJMoa2001017

Zu ZY, Jiang MD, Xu PP, Chen W, Ni QQ, Lu GM, Zhang LJ., Radiology, 200490-200490,2020. https://doi.org/10.1148/ radiol.2020200490 